\title{
Novel Method of Processing IP Traffic in LTE Network
}

\author{
Yuhang Jin1, Hongfei Qi2, Yangyi Yang2 \\ 1Nokia Simens Network, Zhejiang Province, China 2Nokia Simens Network, Zhejiang Province, \\ Chinayuhang-astronee.jin@nsn.com,_Hongfei.qi@nsn.com
}

\begin{abstract}
The central architecture of LTE with IP traffic going though EPC can not assure capacity and performance issue, this paper presents a novel method of processing IP traffic by introducing distribute architecture and using pure IP vendors' technology, removing GTP-U in S1 interface between eNB and EPC. Meanwhile function module and signaling design are introduced. The proposed architecture and method is with advantage of high capacity and performance, easy implementation, while keeping compatible with traditional IP vendor's technology. It is also easy to bring $4 \mathrm{G} \mathrm{LAN}$ concept to LTE technology.
\end{abstract}

Keyword - Liquid Switch, By Pass, Simulation LAN

\section{Introduction1}

Current existing LTE Protocol is designed based on the evolution from $2 \mathrm{G}$ and $3 \mathrm{G}$ technology. As a result, user IP traffic will be encapsulated with GTP-U between eNB and EPC.

This seriously tortures the $4 \mathrm{G}$ vendor's IP process ability in EPC due to a lot of 4G vendors' $\mathrm{eNB}$ and EPC are still based on the $3 \mathrm{G} / 2 \mathrm{G}$ hardware and software platform, obviously, the IP processing is a bottleneck for most of the $4 \mathrm{G}$ vendors bundling the GTP-U traffic between eNB and EPC and brings known problem such as IP capacity and Qos guarantee issue. However, $100 \mathrm{G}, 10 \mathrm{~T}$ is not a problem for vendors like Cisco and Juniper at all, but is very challenging to traditional $3 \mathrm{G}$ vendors and its evolution LTE. Another worse thing is that $4 \mathrm{G}$ vendor can't use the flexible and diversity IP technology if binding up by GTP-U.

Meanwhile, we know, in the 4G world, most of the traffic will be happened between IP terminals, thus majority of IP to IP traffic should not go through EPC. They should go through IP network naturally.

Therefore, in this paper, a novel method of Processing IP traffic is presented by introducing distribute architecture and using pure IP vendors' technology, by removing GTP-U in S1 interface between eNB and EPC. The Novel architecture and Method has the advantages of fulfill capacity and performance requirement, good compatibility to traditional IP vendor technology, easy implementation with litter modification in current LTE signaling system.

\section{Novel Method of Processing IP Traffic}

\section{Review of Previous LTE Protocol Architecture}

In previous architecture Ref. [123] in 3GPP, an EPS bearer has to cross multiple interfaces as shown in Fig.1 with the $\mathrm{S} 5 / \mathrm{S} 8$ interface from the P-GW to the S-GW, the S1 interface from the S-GW to the eNB, and the radio interface from the eNB to the UE. Within each interface, the EPS bearer is mapped into a lower layer bearer, each with its own bearer identity and label. Each node keep track of the binding between the bearer IDs across its different interfaces.

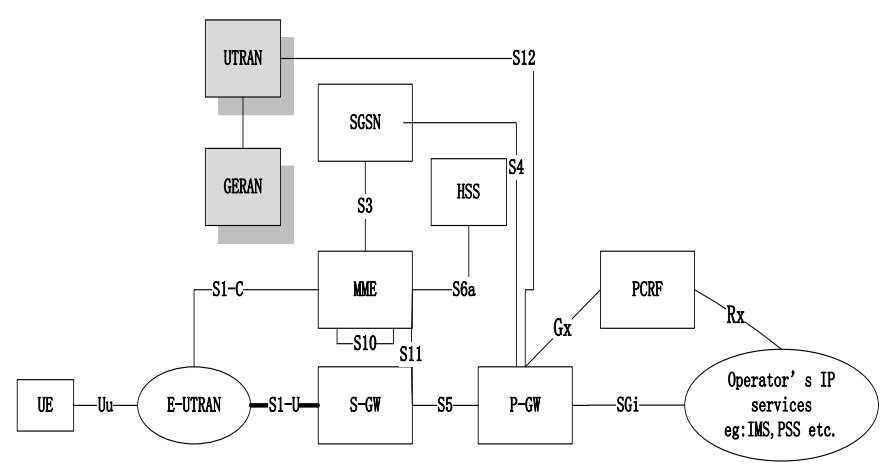

Fig.1. Previous LTE Protocol architecture

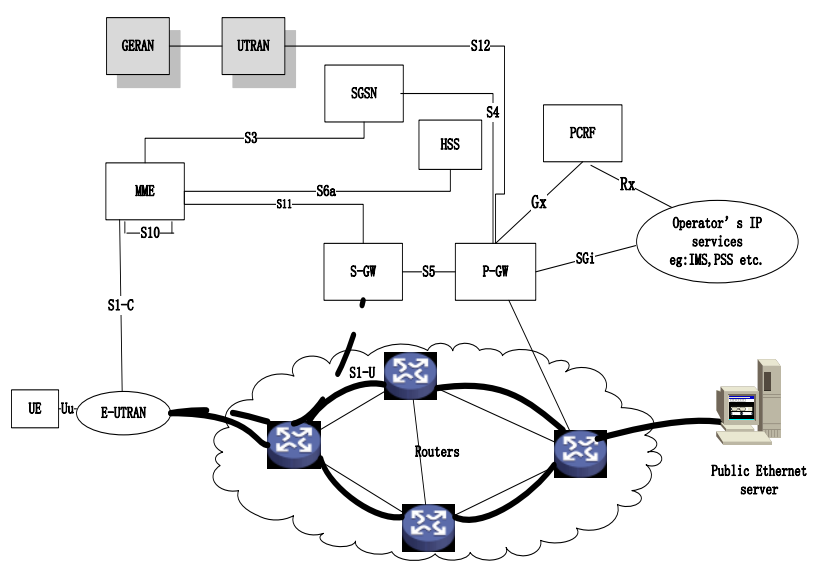

Fig.2. New overall traffic architecture

In our new architecture for User Plane data as shown in Fig.2, an EPS bearer does not need to cross multiple interfaces as result of removing the S5/S8 interface from the P-GW to the $\mathrm{S}-\mathrm{GW}$, the $\mathrm{S} 1$ interface from the S-GW to the eNB from data path.

Thus, in the downlink direction, IP packet from IP network will route to eNB directly. And eNB indexes the bear though the Packet filtering function of using IP header information such as source and destination IP addresses. The Uplink direction processes in the similar way.

\section{Requirement and Principle}

Targeting on by pass IP traffic, meanwhile keeping IP stream still controllable and compatible. The following is technology proposals: 
Add new module called Liquid Switch in eNB, MME, SGW, PGW network elements. The new modes should support specific kind U-plane ne traffic packet switch back and forth between GTP-U and general Ethernet router/switch which connects to public IP router directly.

Add new singling to guarantee new added modules works well and compatible with the standard 3GPP specification.

Liquid Switch is a network optimization of current LTE network, by using this new module, the $4 \mathrm{G}$ user IP-to-IP traffic was forwarded directly to next hop router and data traffic is off-load from EPC.

The Liquid Switch Mode should support both IPv4 and IPv6 mode with IP security function.

eNB should support dual IP encapsulation Protocol stack: IP Protocol stack without GTP-U called in Liquid Switch Mode and GTP-U for U-plane data called in Legacy Mode. The two protocol stack should be coexisting in eNB.

The Liquid Switch Mode can be configured in system level manually or in auto smart algorithm mode. The system relies on use smart algorithm and UE associated signaling for the Liquid Switch Mode control, which is either streaming based or UE based. The smart logic means system can select legacy/streaming based Liquid Switch/UE based Liquid Switch depend on some pre-condition configuration like cell loading, EPC loading etc.

Both EPC and eNB should be responsible for the smart algorithm execution and UE associated signaling; EPC should have the central role and final decision about the Liquid Switch Mode control.

In both EPC and eNB, UE Liquid Switch state machine should been added to record the detail of current mode.

The implementation of Liquid Switch must not remove any old functionality from the LTE/SGW architecture, and guarantee compatible to 3GPP standard. Liquid Switch should also add some new Signaling, billing, Qos, Security mechanism.

In deploying the Liquid Switch, the IPRAN network assignment and plan may be impacted. In one word, need to take UE IP assignment as part of IPRAN to guarantee the IP routing switch can reach IP world. Vlan is as an option based on the network design, if it's L2 network.

The traffic between eNB and intra eNB can go through Liquid Switch; Liquid Switch may bring benefit for $4 \mathrm{G}$ simulation LAN function.

\section{Liquid Switch Function Module Design}

Add new function module in EPC and eNB to support Liquid Switch:

\section{Liquid Switch Sub-function Module in EPC}

Smart control algorithm: this module decides when and how system triggers and executes the Liquid Switch strategy, which is the overall control module on EPC;

Initial configuration: Liquid Switch related parameters and strategy configuration during the system startup;

UE state machine: maintain UE Liquid Switch state machine;
Liquid Switch control signaling: UE associated signaling on EPC, including liquid link establishment, liquid link release, liquid link detection and exception handling;

Statistics and charging: gather the statistics reported from eNB and generate charging information in Liquid Switch Mode.

Liquid Switch Sub-function Module in eNB

Smart control algorithm: this module decides when and how system triggers or executes the Liquid Switch strategy, which is the overall control module on eNB;

Initial configuration: Liquid Switch related parameters and strategy configuration when the system startup;

$U E$ state machine: maintain UE Liquid Switch state machine;

Liquid Switch control signaling: UE associated signaling on eNB NE, including liquid link response to MME's request, liquid link release, liquid link detection and exception handling;

IP statistics reporting: gather the statistics reported and send it to EPC, either period based or event based;

\section{Procedures and Signaling}

EPS Bearer link Establishment in Liquid Switch

EPS Bearer Liquid Switch link Establishment contains the operations and procedures for establishing Liquid Switch for EPS bear(s). It consists of signaling and data radio bearer establishment procedures. The procedure is triggered by EPC side from MME /SGW/ PGW.

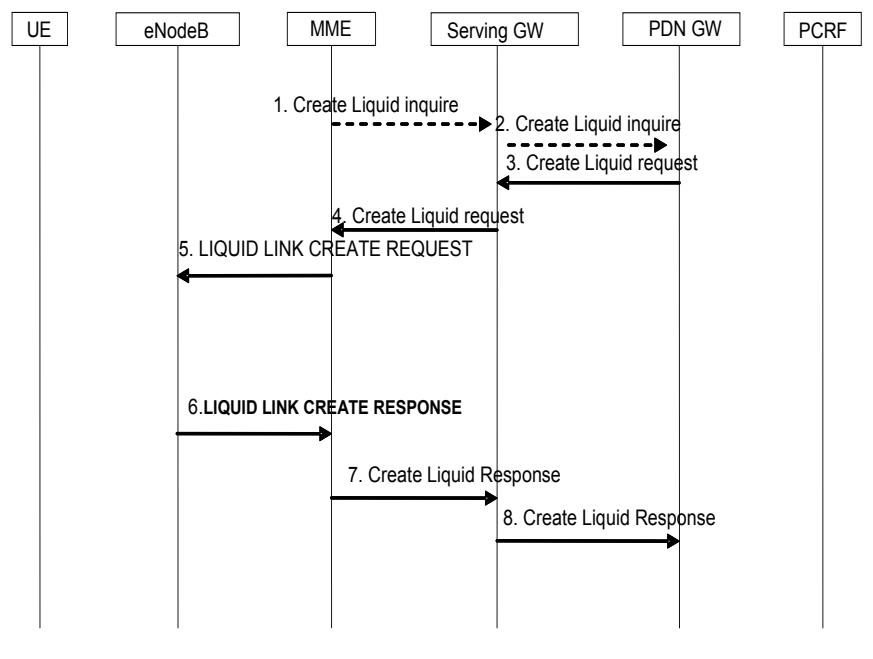

Fig.3. Liquid Switch establish overall procedure

EPS Bearer llink Modification in Liquid Switch

EPS Bearer Liquid Switch link Modification contains the operations and procedures for modify Liquid Switch for EPS bear(s). It consists of signaling and data radio bearer modification procedures. The procedure is triggered by EPC side from MME /SGW/ PGW. 


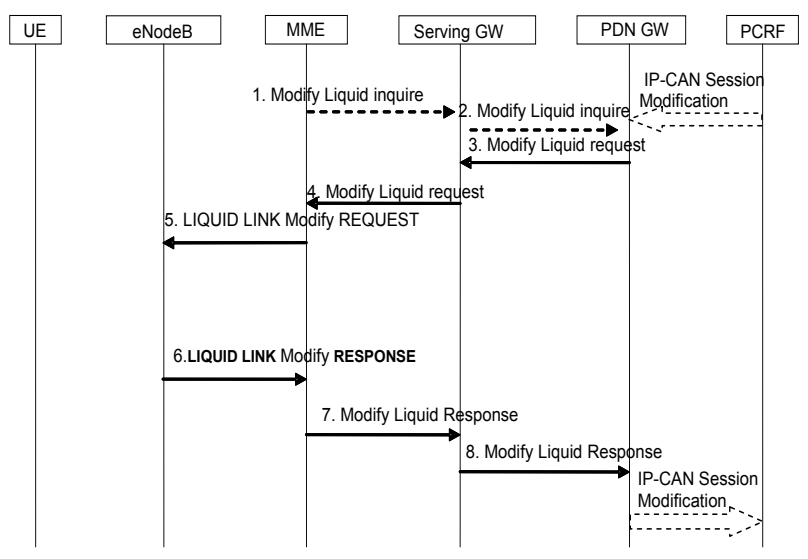

Fig.4. Liquid Switch modify overall procedure

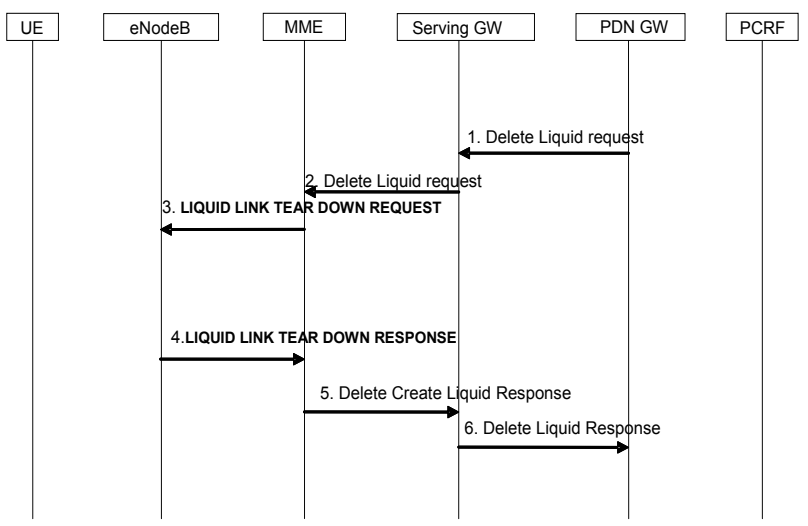

Fig.5. PGW initiated Liquid Switch link release overall procedure

\section{EPS Bearer Liquid Switch link Release}

EPS Bearer Liquid Switch link Release contains the operations and procedures for Release Liquid Switch for EPS bear(s). It consists of signaling and data radio bearer Release procedures.

The procedure is triggered by EPC side from MME /SGW/ PGW or eNB side.

\section{Simulation LAN through LTE}

Currently all $4 \mathrm{G}$ user data forwarding can only through X2 between in case of intra-eNB, and even S1 link is still needed if there is no $\mathrm{X} 2$ connection is for neighbor eNB.

In Liquid Switch module, UE streaming was encapsulated as standard IP packets, which means, in the data plane, a group of eNB can be considered as a small scale LAN, which can forward data in IP nature behavior through interworking with connected IP Switchers and no needs to be processed by SGW or PGW again by tunnel. It reduces the data latency, and also improves the data rate and lease the $4 \mathrm{G}$ network burden. We call it simulation LAN. Of course, all the UE traffic status is still monitored through EPC by the liquid heart beat messages.

As LTE is targeting on the wireless broadband IP network solution, the user traffic should still on 80/20 (local/internet) rule, and lots of date will come from local. So it is very easy to bring simulation LAN concept to LTE with this novel method of processing of IP traffic
Similar to normal Ethernet network, there are two kinds of VLAN mode, Static and Dynamic. Static VLAN is eNB based VLAN, while dynamic is User based by IMEI number.

Static Mode

In static mode, the Ethernet switch network topology was planned and configured during initial configuration stage. Usually the VLAN is bound with eNB physical uplink interface, when any UE comes into liquid switch mode; eNB will add the pre-defined VLAN tag to the data.

1) Intra eNB with Same VLAN

Step 1: in Fig.6, UE attach to EPC then switch to liquid switch module after MME's command;

Step2: Source eNB received the data. according to the destination IP, source eNB recognized that the target IP is an internal IP;

Step3: eNB Forward data directly;

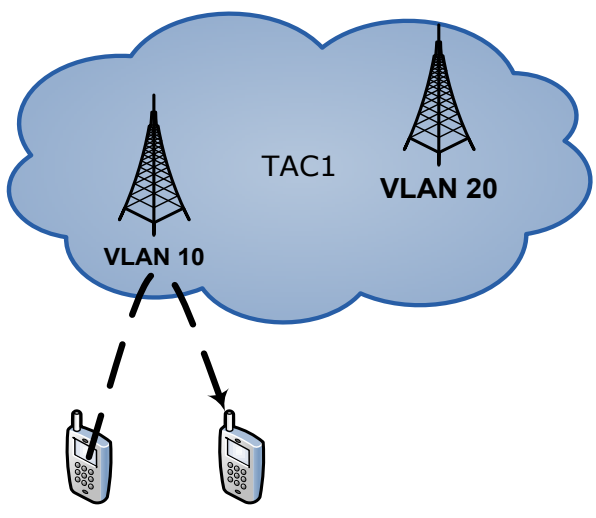

Fig.6. Intra eNB data forward with the same VLAN

2) Inter eNB via $X 2$ Llink with Same VLAN

Step 1: in Fig.7,UE attach to EPC then switch to liquid switch module after MME's command;

Step 2: Source eNB will check the X2 link status between the source and target eNB. If the X2 is available, it's an option policy configured by operators to decide whether use $\mathrm{X} 2$ as a highly preference.

Step 3:Target eNB received the data from X2 link, forward them to target UE.

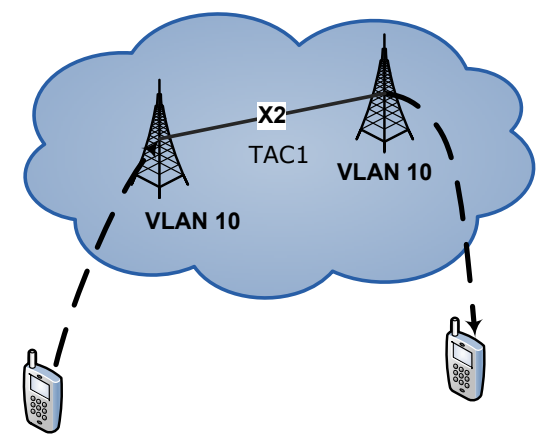

Fig.7. Inter eNB data forward via $\mathrm{X} 2$ 
3) Inter eNB via Ethernet Switch with Same VLAN

Step 1: UE attach to EPC then switch to liquid switch module after MME's command;

Step 2: Source eNB received the data. According to the destination IP, source eNB recognized that the target IP is not an eNB internal IP, so it will forward the data to default gateway. Before the data send out, it should obtain the next hop IP address;

Step3: Source eNB will check its ARP table; found that the destination IP belongs to VLAN 10 too, so it will forward the data directly by target MAC address.

Step4: Destination eNB received the data, and move the VLAN tag and forward data to the destination UE.

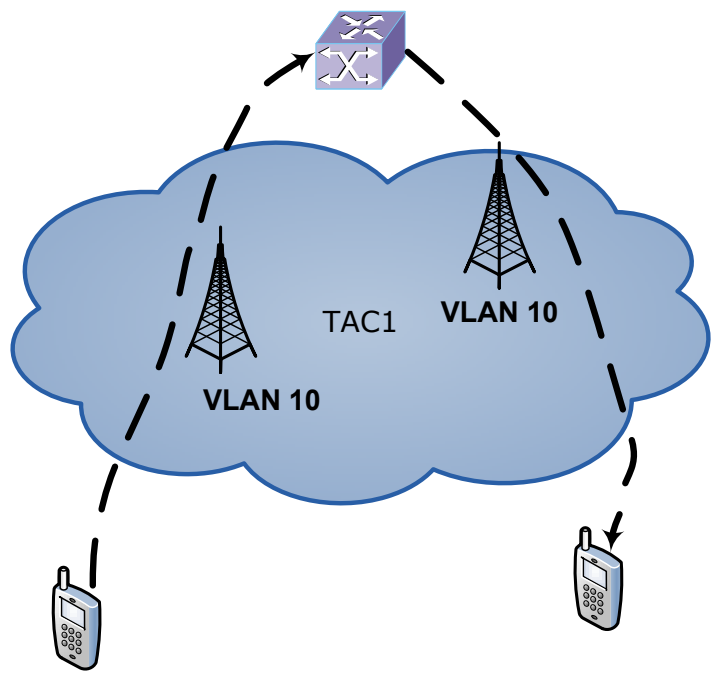

Fig.8. Inter eNB data forward via Ethernet switch with the same VLAN

4) Inter eNB via Ethernet switch with Different VLANs

Step 1: UE attach to EPC then switch to liquid switch module after MME's command;

Step 2: Source eNB received the data. According to the destination IP, source eNB recognized that the target IP is not an eNB internal IP, so it will forward the data to default gateway. Before the data send out, it should obtain the next hop IP address and the data will be tagged VLAN 10;

Step3: Source eNB will check its ARP table; found that the destination IP can't found in VLAN10, then forward the data to $\mathrm{L} 3$ route module.

Step4: The L3 route module in eNB will check the route table, found that the target IP is in VLAN 20, accordingly add VLAN 20 tag to the data and then forward them to VLAN 20. Target eNB received the data, and remove the VLAN tag and forward data to target UE.

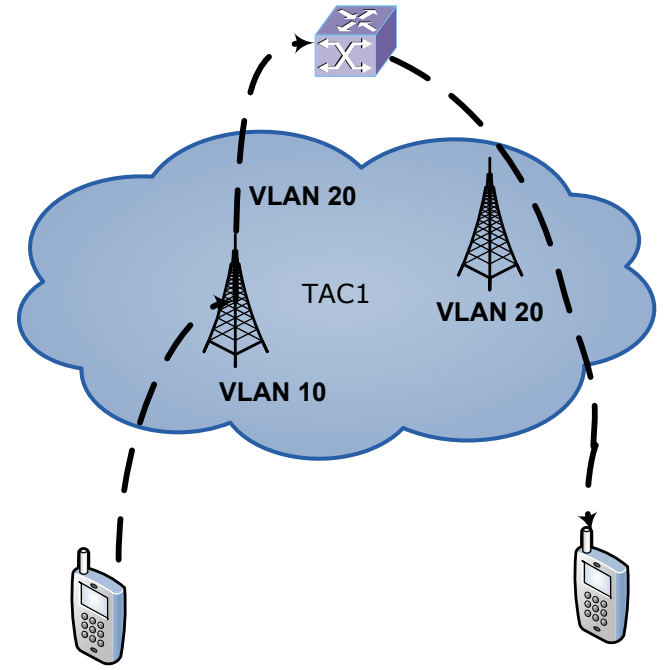

Fig.9. inter eNB data forward via Ethernet switch with different VLANs

\section{Dynamic Mode}

There are increasing demand for the virtual LAN for the L1/L2 traffic isolation and user security, we will have the similar conception in the LTE virtual LAN simulation.

In dynamic mode, VLAN tag is not based on the physical eNB Vlan configuration anymore but based on UE's Identity or group. When UE's data reach eNB, it will be add a certain VLAN tag based on the user data, and the other traffic forward behavior is similar to static mode. The difference is that once an UE is bound to one VLAN, its data will always be tagged the same tag wherever the eNB the UE located.

In this mode, eNB should support trunk vlan function, and dynamically builds the vlan table in the eNB uplink with Cisco/Juniper switches etc.

\section{Conclusion}

In this paper, a novel kind of protocol architecture and IP processing method without GTP-U are proposed to fulfill capacity and performance requirement. The Liquid switch overall system requirements, function module and signaling design is introduced. The proposed solution helps to solve the bottleneck for most of the $4 \mathrm{G}$ vendors within their core network. Meanwhile with Liquid switch, it is easy to bring $4 \mathrm{G}$ LNA concept into LTE network.

\section{References}

[1] Sassier, I.Toufik, M.baker, LTE The UMTS Long Term Evolution,Wiley, 2009, pp24-12

[2] 3GPP, 3GPP TS 36.300 V8.7.0 (2008-12), pp.15-21

[3] 3GPP, 3GPP TS 23.401 V10.0.0 (2010-06), pp.14-13 